\title{
Communication \\ A Multiplicative Regularizer Augmented with Spatial Priors for Microwave Imaging
}

\author{
Nozhan Bayat, Student, IEEE and Puyan Mojabi, Member, IEEE
}

\begin{abstract}
The standard weighted $L_{2}$ norm total variation multiplicative regularization (MR) term originally developed for microwave imaging algorithms is modified to take into account structural prior information, also known as spatial priors (SP), about the object being imaged. This modification adds one extra term to the integrand of the standard MR, thus, being referred to as an augmented MR (AMR). The main advantage of the proposed approach is that it requires a minimal change to the existing microwave imaging algorithms that are already equipped with the MR. Using two experimental data sets, it is shown that the proposed AMR (i) can handle partial (incomplete) SP, and (ii) can, to some extent, enhance the quantitative accuracy achievable from microwave imaging.
\end{abstract}

Index Terms-Microwave imaging (MWI), regularization, spatial priors (SP), inversion.

\section{INTRODUCTION}

$\mathbf{M}$ ICROWAVE imaging (MWI) is a non-invasive imaging method with which quantitative images of the relative complex permittivity profiles of the objects of interest (OI) can be created. This is often done by applying an electromagnetic inverse scattering algorithm (or, simply an inversion algorithm hereafter) to the microwave scattered field data collected outside the OI. In some situations, the measured data might not have sufficient information content to reconstruct the OI to the desired accuracy. In such cases, one option is to provide the inversion algorithm with some prior information. This Communication is focused on a method to inject prior structural information, also known as spatial priors (SP), into the inversion algorithm. These SP may come from a different higher-resolution imaging modality such as magnetic resonance imaging (MRI) [1] or ultrasound imaging [2], [3]. For example, in [4], an image registration approach was proposed to use MRI images in MWI reconstruction. In addition, a combined imaging technology named magnetic resonance microwave tomography was proposed in [1]. Instead of using SP in MWI reconstruction, another approach is to perform joint inversion of electromagnetic data with another type of data such as acoustic data to improve the achievable image accuracy [5]. The joint inversion of such multi-physics data can be, for example, linked through appropriate similarity regularization terms such as cross-gradient functions [6]. The joint inversion is not within the scope of this paper.

This paragraph of the first footnote will contain the date on which you submitted your paper for review. The authors acknowledge Natural Sciences and Engineering Research Council (NSERC) of Canada, and Canada Research Chair Program for their financial support.

The authors are with the Department of Electrical and Computer Engineering at the University of Manitoba, Winnipeg, MB, Canada (email: Puyan.Mojabi@UManitoba.ca).
Incorporation of SP into MWI inversion algorithms has already been considered by several authors using different approaches [1] , [2], [7]-[15]. These methods can be classified (at least) under three categories: (i) those that favour similarity of the reconstructed permittivity values in each region within the SP, which we refer to as similarity-based approaches, e.g., [9], (ii) those that use an inhomogeneous background medium derived from the SP for the inversion algorithm, e.g., [13], and (iii) those that use special basis functions obtained based on the SP for the expansion of the unknown permittivity profile in the inversion process, e.g., [12]. The proposed algorithm in this paper enforces SP in a different manner: via providing edge information for the inversion algorithm based on the SP. (An edge represents the transition from one region to another in the complex permittivity image.) We refer to this type of SP algorithms as edge-based approaches. Noting that edges are associated with the gradient of the image, edge-based approaches are somehow related to similarity-based approaches through the gradient operator. It should be noted that the proposed edge-based algorithms can retrieve the permittivity of those regions that are absent in the incomplete SP. (That is, in such regions, these SP algorithms switch back to the regular inversion.) This is an indication that the proposed edge-based approach belongs to the class of soft prior regularization as opposed to hard prior regularization [8].

The main feature of this algorithm is that it only requires a minimal change to the existing Gauss-Newton inversion (GNI) and contrast source inversion (CSI) algorithms equipped with the standard multiplicative regularizer (MR), e.g., [16], [17]. (The term 'standard' indicates the common MR used in the literature: the weighted $L_{2}$ norm total variation multiplicative regularizer, e.g., see [17].) Using the proposed SP technique, the existing MR-GNI and MR-CSI algorithms can be easily changed to accommodate the available SP by augmenting the integrand of the standard MR by an extra term. Thus, we refer to it as an augmented MR (AMR). When the proposed AMR is used with GNI and CSI, we refer to the resulting algorithms as AMR-GNI and AMR-CSI respectively. Herein, we first briefly review the standard MR, and then describe the small change that needs to be done in order to incorporate SP into the existing MR. We then evaluate the performance of AMR-GNI and AMR-CSI against two experimental data sets for two-dimensional scalar microwave imaging. In addition, we compare the performance of the proposed algorithms against a different SP algorithm, entitled MRSP-GNI [14]. The MRSP-GNI algorithm belongs to the class of similarity-based approaches. This is in contrast to the AMR-GNI and AMRCSI algorithms, which are edge-based approaches. 


\section{Multiplicative Regularizer (MR) - Review}

The MR-GNI and MR-CSI algorithms use the following multiplicative regularization term [17]

$$
\mathcal{C}_{n}^{\mathrm{MR}}(\chi)=\frac{1}{A} \int_{D} \frac{|\nabla \chi(\mathbf{r})|^{2}+\delta_{n}^{2}}{\left|\nabla \chi_{n}(\mathbf{r})\right|^{2}+\delta_{n}^{2}} d \mathbf{s}
$$

to regularize their associated cost functionals at the $n$th iteration of the algorithms. In (1), $A$ is the area of the imaging domain $D, \chi$ is the unknown relative complex permittivity contrast profile, $\chi_{n}$ is the known estimate of $\chi$ at the $n$th iteration, $\mathbf{r}$ is the position vector spanning the imaging domain, $\nabla$ is the gradient operator, and $|\cdot|$ is the magnitude operator. The steering parameter $\delta_{n}^{2}$, which is a real number, gets smaller as the inversion algorithm gets closer to the final solution. (For the expressions of $\delta_{n}^{2}$ in the MR-GNI and MR-CSI algorithms, see [16] and [17], respectively.) The operation of MR has been described in previous works such as [17]-[19]; herein, we only provide a quick overview. To this end, let us consider the gradient operator of the MR at the $n$th iteration of the algorithm that operates on a given $\chi$ [20, Appendix D.3]

$$
\mathcal{L}_{n}^{\mathrm{MR}}(\chi)=-\nabla \cdot\left(b_{n}^{2}(\mathbf{r}) \nabla \chi\right)
$$

where ' $\nabla$ ' ' is the divergence operator, and $b_{n}^{2}(\mathbf{r})$ is an inhomogeneous weight given as

$$
b_{n}^{2}(\mathbf{r})=\frac{1}{A} \frac{1}{\left|\nabla \chi_{n}(\mathbf{r})\right|^{2}+\delta_{n}^{2}} .
$$

In the first few iterations, $\delta_{n}^{2}$ is dominant as compared to $\left|\nabla \chi_{n}(\mathbf{r})\right|^{2}$ at most positions $\mathbf{r}$. Therefore, in early iterations, $b_{n}^{2}(\mathbf{r})$ can be approximated by the constant number $1 /\left(A \delta_{n}^{2}\right)$. Consequently, in the early iterations, we have

$$
\mathcal{L}_{n}^{\mathrm{MR}}(\chi) \stackrel{\text { Early iterations }}{\approx} \frac{1}{A \delta_{n}^{2}} \nabla \cdot(\nabla \chi)=\frac{1}{A \delta_{n}^{2}} \nabla^{2} \chi
$$

where ' $\nabla^{2}$ ' is the Laplacian operator. That is, in early iterations, MR works similar to Laplacian regularization which aims to smoothen the reconstruction. After early iterations, the relative magnitude of $\left|\nabla \chi_{n}(\mathbf{r})\right|^{2}$ and $\delta_{n}^{2}$ should be considered locally in the imaging domain. If in a region within the imaging domain, $\delta_{n}^{2}$ is dominant compared to $\left|\nabla \chi_{n}(\mathbf{r})\right|^{2}$, the above Laplacian approximation is still valid locally, and the regularization operator then attempts to smooth out that region. Otherwise, it favours to reconstruct an edge in that region.

\section{Augmented Multiplicative Regularizer (AMR)}

Let us now consider that we have some available SP about the OI. Given the SP, we can then guide the inversion algorithm regarding the locations at which some edges (i.e., the boundaries between different regions) are to be expected. To this end, we not only rely on the relative magnitude of $\left|\nabla \chi_{n}(\mathbf{r})\right|^{2}$ and $\delta_{n}^{2}$ for edge detection as in (1), but we also introduce an extra term based on the available SP to further guide the inversion algorithm in edge detection. Thus, we modify the MR given in (1) to an augmented MR (or, simply AMR) as follows

$$
\mathcal{C}_{n}^{\mathrm{AMR}}(\chi)=\frac{1}{A} \int_{D} \underbrace{\frac{|\nabla \chi(\mathbf{r})|^{2}+Q^{2}|\nabla P(\mathbf{r})|^{2}+\delta_{n}^{2}}{\left|\nabla \chi_{n}(\mathbf{r})\right|^{2}}+\underbrace{Q^{2}|\nabla P(\mathbf{r})|^{2}}_{\text {Edge II }}+\delta_{n}^{2}}_{\text {Edge I }} d \mathbf{s} .
$$

As can be seen from (5), we have now augmented the integrand of the MR with an extra term, $Q^{2}|\nabla P(\mathbf{r})|^{2}$, where $P$ incorporates SP. In our implementation, $P$ is a matrix containing some numbers, each of which represents an expected region within the imaging domain $D$. For example, if the SP contain four regions, we assign the values of $1,0.75,0.50$, and 0.25 to each of these regions 1 In addition, $Q$ is a scaling factor that controls the relative weight of $|\nabla P|^{2}$. In all the examples shown in this Communication, this scaling factor has been set to $Q=1$. Also, in (5), 'Edge I' refers to the ability of this regularizer to retrieve edges from the reconstructed contrast at the previous iteration, and 'Edge II' refers to the ability of this regularizer to retrieve edges from the provided SP. One question that may arise is the following: why do we use both $\left|\nabla \chi_{n}(\mathbf{r})\right|^{2}$ and $|\nabla P(\mathbf{r})|^{2}$ in the AMR? The main reason lies in the fact that if the SP only provide partial (incomplete) structural information, the presence of $\left|\nabla \chi_{n}(\mathbf{r})\right|^{2}$ may still enable the inversion algorithm to retrieve the edges that are not present in the SP. In addition, if some of the edges provided by the SP are wrong, the presence of $\left|\nabla \chi_{n}(\mathbf{r})\right|^{2}$ may alleviate the effects of the wrong edges to some extent. Similar to the $\mathrm{MR}$, the gradient of the AMR at the $n$th iteration will be

$$
\mathcal{L}_{n}^{\mathrm{AMR}}(\chi)=-\nabla \cdot\left(a_{n}^{2}(\mathbf{r}) \nabla \chi\right)
$$

where

$$
a_{n}^{2}(\mathbf{r})=\frac{1}{A} \frac{1}{\left|\nabla \chi_{n}(\mathbf{r})\right|^{2}+Q^{2}|\nabla P(\mathbf{r})|^{2}+\delta_{n}^{2}} .
$$

The operator $\mathcal{L}_{n}^{\mathrm{AMR}}$ works similarly as $\mathcal{L}_{n}^{\mathrm{MR}}$ with only one difference: in addition to the relative magnitude of $\left|\nabla \chi_{n}(\mathbf{r})\right|^{2}$ and $\delta_{n}^{2}$, we now have an extra term, $Q^{2}|\nabla P(\mathbf{r})|^{2}$, that also plays a role in edge reconstruction. Also, note the simplicity of incorporating SP within the existing MR-GNI and MRCSI algorithms. Herein, the only modification performed was to replace $b_{n}^{2}$ given in 3 by $a_{n}^{2}$ given in (7). Upon this replacement, the two algorithms are referred to as AMR-GNI and AMR-CSI.

Finally, let us address this question: what happens if we remove $\left|\nabla \chi_{n}\right|^{2}$ from the denominator of (5)? That is, what happens when we convert AMR to the so-called AMR (Type II) as

$$
\mathcal{C}_{n}^{\text {AMR-II }}(\chi)=\frac{1}{A} \int_{D} \frac{|\nabla \chi(\mathbf{r})|^{2}+\delta_{n}^{2}}{Q^{2}|\nabla P(\mathbf{r})|^{2}+\delta_{n}^{2}} d \mathbf{s} .
$$

As expected the edges are now merely determined by the relative magnitude of $Q^{2}|\nabla P(\mathbf{r})|^{2}$ and $\delta_{n}^{2}$. Let us assume that there is no available prior edge information in certain regions within the imaging domain; then, $|\nabla P(\mathbf{r})|$ will be zero in those regions. Thus, similar to (4), the regularization operator

\footnotetext{
${ }^{1}$ In this paper, assuming $N$ regions, we start by assigning 1 to a given region, then $1-\frac{1}{N}$ is assigned to another region, and finally $1-\frac{N-1}{N}$ is assigned to the last region. The assignment of these numbers to different regions is ad hoc. Based on our numerical experience, the performance of the proposed algorithm is not very sensitive to the order by which we assign these numbers to different regions. Also, the reason behind choosing the maximum value of $P$ to be one is based on assuming that a typical maximum contrast value in MWI (assuming the presence of a high permittivity matching fluid) is about one. Thus, throughout this paper, the maximum value of $P$ was set to one in an attempt to make $\left|\nabla \chi_{n}(\mathbf{r})\right|^{2}$ and $|\nabla P(\mathbf{r})|^{2}$ relatively balanced in 5 . (This is despite the fact that the maximum contrast value in the imaging domain is 2 in the first example considered in this paper.)
} 


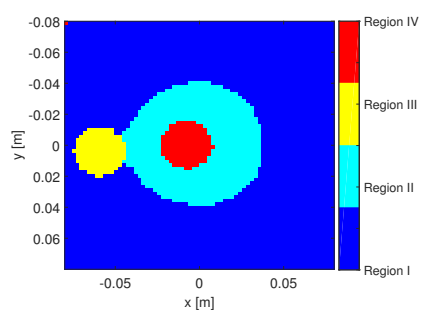

(a) Complete SP

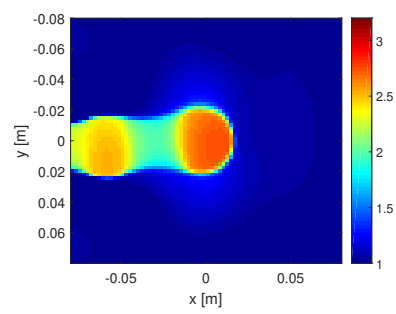

(c) MR-GNI (blind)

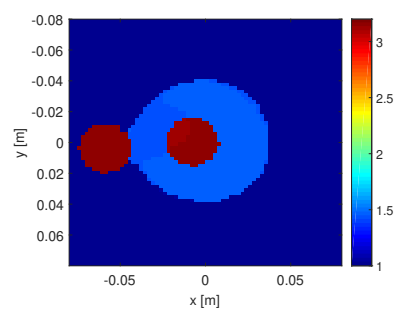

(e) MRSP-GNI

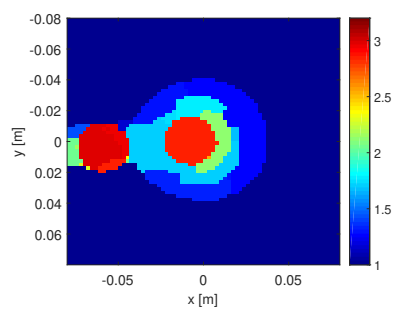

(g) AMR-CSI (b) $|\nabla P|^{2}$

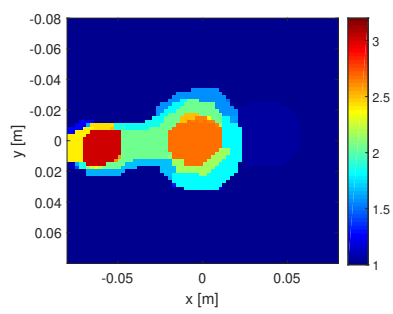

(d) MR-CSI (blind)

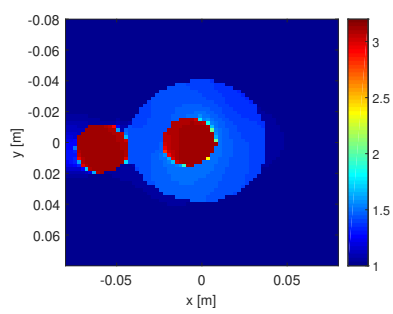

(f) AMR-GNI

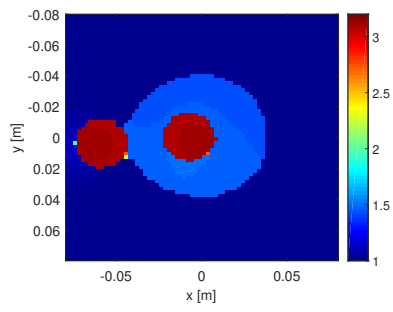

(h) AMR-CSI (Type II)

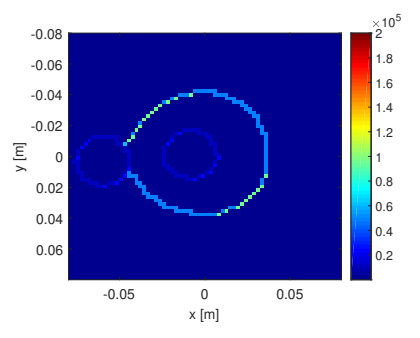

Fig. 1. (a) The SP showing four regions of the FoamTwinDielTM target of Institut Fresnel. (b) The SP in the form of edges $\left(|\nabla P|^{2}\right)$ to be given to the AMR-based algorithms. The reconstructed real part of the relative complex permittivity at $2 \mathrm{GHz}$ using (c) MR-GNI, (d) MR-CSI, (e) MRSPGNI, (f) AMR-GNI, (g) AMR-CSI, and (h) AMR-CSI (Type II).

switches to Laplacian operator in such regions. In summary, if we use (5), edge-preserving might still be applied to the regions that are absent in the incomplete SP. However, if we use (8), Laplacian operator will be applied to such regions. We refer to the use of $(8)$ as $A M R$ (Type II) ${ }^{2}$

\section{EXPERIMENTAL RESUlts}

We consider two previously-reported experimental data sets to evaluate the performance of the AMR-GNI and

${ }^{2}$ In [5], 21], the standard MR was modified to allow for the joint inversion of acoustic and electromagnetic data. To this end, the gradient of the acoustic contrast is used for edge detection in electromagnetic inversion and vice versa. This joint MR has a similar structure as AMR (Type II) considered herein.
AMR-CSI algorithms. The first data set is the so-called FoamTwinDielTM data set [22] collected by the Fresnel Institute in an anechoic chamber. The second data set was collected by the Electromagnetic Imaging Laboratory at the University of Manitoba from a human forearm [16] in the matching fluid of salt water. In all the cases presented below, all the inversion algorithms start with a trivial initial guess, which is the zero contrast for the AMR-GNI algorithm, and the back-propagated solution for the AMR-CSI algorithm.

\section{A. FoamTwinDielTM Data Set}

This target consists of three dielectric cylinders, two of which have a diameter of $31 \mathrm{~mm}$, and a relative permittivity of $\epsilon_{r}=3 \pm 0.3$ and the other one has a diameter of $80 \mathrm{~mm}$ and a relative permittivity of $1.45 \pm 0.15$. The geometry of this target can be found in [22]. Herein, we have set the size of the imaging domain to $16 \times 16 \mathrm{~cm}^{2}$ discretized into $70 \times 70$ square cells. This target was illuminated from 18 different angles, and the resulting scattered field are collected at 241 data points per transmitter. (This procedure has been repeated at 9 different operational frequencies ranging form $2 \mathrm{GHz}$ to $10 \mathrm{GHz}$ with $1 \mathrm{GHz}$ increment.) Similar to the procedure outlined in [23], we have created the SP shown in Figure 1 (a) for this target. However, note that the utilized SP in the proposed AMR approach is in the form of preferred edges through $|\nabla P|^{2}$ as shown in Figure 1 (b). The scale of $|\nabla P|^{2}$ can be understood by noting that the gradient operator involves division by $\Delta x$ and $\Delta y$, each of which represents the size of a discretized cell within the imaging domain along the $x$ and $y$ directions respectively.

Let us now consider the inversion at the lowest frequency of operation, i.e., $2 \mathrm{GHz}$. The reason for this intentional choice of this frequency is for the blind ${ }^{3}$ inversion using MR-GNI and MR-CSI to not yield sufficient resolution; see Figure 1(c)(d). (These blind reconstructions have already been shown by several groups including the authors, e.g., [23], and are only presented herein for the sake of side-by-side comparison with the inversion using the SP.) Incorporating the SP through the similarity-based approach using the MRSP-GNI algorithm [14] is shown in Figure 11(e), which shows a high quantitative accuracy. Incorporating the SP in the form of preferred edges using the AMR-GNI and AMR-CSI algorithms results in the reconstructions shown in Figure $1 \mathrm{f})-(\mathrm{g})$. As can be seen, the AMR-GNI almost perfectly reconstructs the relative permittivities of the dielectric objects. In addition, the AMR-CSI reconstructed values, although not perfect, are still better than those obtained by MR-CSI. From our numerical experience, the use of AMR seems to work better with the GNI algorithm as compared to the CSI algorithm. By noting the presence of spurious edges in the MR-CSI (blind) reconstruction, see Figure 1/d), the relatively poor performance of the AMR-CSI algorithm could be due to the common factor of MR-CSI and AMR-CSI, i.e., $\left|\nabla \chi_{n}\right|^{2}$. To investigate this, we have tried inverting this data set with AMR-CSI (Type II) which removes $\left|\nabla \chi_{n}\right|^{2}$ from its regularizer. As can be seen in Figure 1(h), the

\footnotetext{
${ }^{3}$ The term blind inversion indicates that no prior information has been used in the inversion process.
} 


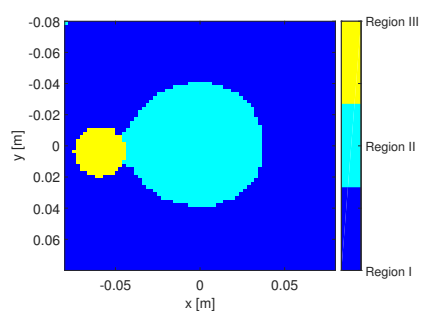

(a) Incomplete (partial) SP

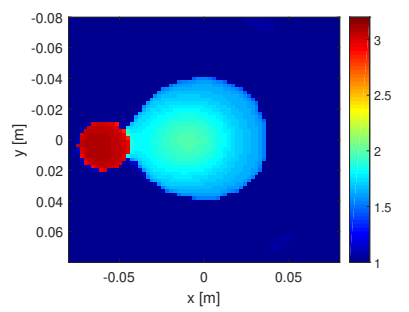

(c) MRSP-GNI

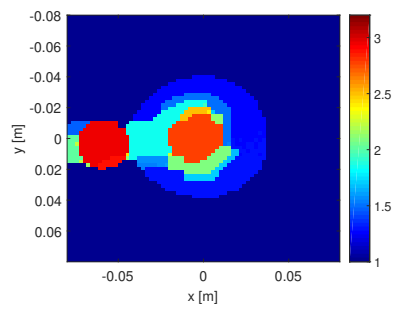

(e) AMR-CSI

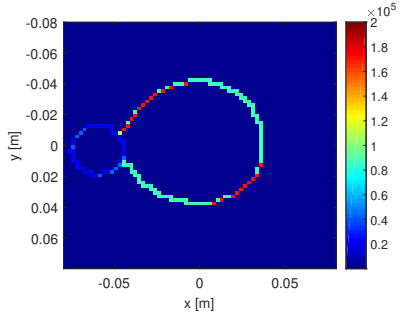

(b) $|\nabla P|^{2}$

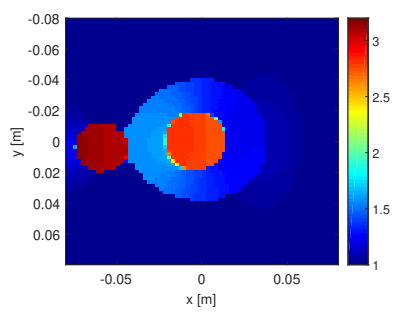

(d) AMR-GNI

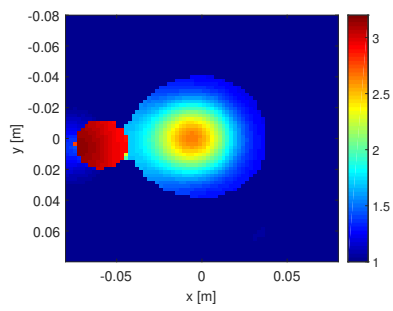

(f) AMR-CSI (Type II)
Fig. 2. (a) The incomplete (partial) SP for the FoamTWinDielTM target showing three regions within the imaging domain with the inner cylinder missing from the SP. (b) The partial SP in the form of edges. The reconstructed real part of the relative complex permittivity at $2 \mathrm{GHz}$ using (c) MRSP-GNI, (d) AMR-GNI, (e) AMR-CSI, and (f) AMR-CSI (Type II).

AMR-CSI (Type II) reconstruction is almost perfect, which is aligned with our speculation $4^{4}$ In the next step, we only provide partial (incomplete) SP to the inversion algorithms. The partial SP are illustrated in Figure 2 (a) where it is clear that the SP have no knowledge of the inner dielectric cylinder. The SP in the form of preferred edges is shown in Figure 2 2 b) in which the edges associated with the inner cylinder are missing. The reconstruction results of the MRSP-GNI, AMR-GNI, AMRCSI, and AMR-CSI (Type II) are shown in Figures 2(c)-(f). As can be seen, the MRSP-GNI algorithm (a similarity-based approach) was not able to fully retrieve the inner dielectric cylinder as it was misguided by the partial $\mathrm{SP}{ }^{5}$ However, both AMR-GNI and AMR-CSI are successful in the detection of

\footnotetext{
${ }^{4}$ We have also tried AMR-GNI (Type II); it provided similar reconstruction as in AMR-GNI but it required tuning $Q$ parameter in AMR (Type II) to be 10. For brevity, this is not shown here.

${ }^{5}$ It should be noted that MRSP-GNI is, in fact, able to retrieve the inner cylinder if it is told that the available SP is only partial and is not accurate within the larger cylinder. As discussed in [14], the mechanism with which we can tell the MRSP-GNI algorithm that a given SP is partial is via the use of a probability vector. Herein, we have not used this probability vector Subsequently, MRSP-GNI favours similar permittivity values within Region II of Figure 2 a), thus, missing the inner cylinder. On the other hand, in [23], the MRSP-GNI algorithm was told that the SP within Region II is not accurate, thus, we were able to reconstruct the inner cylinder using MRSP-GNI.
}

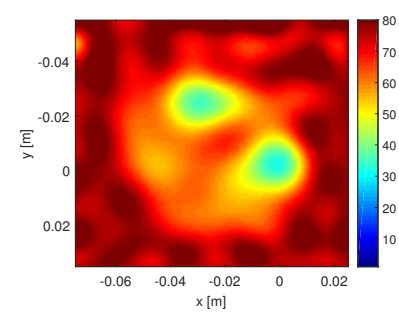

(a) $\operatorname{Re}\left(\epsilon_{r}\right)$, MR-GNI

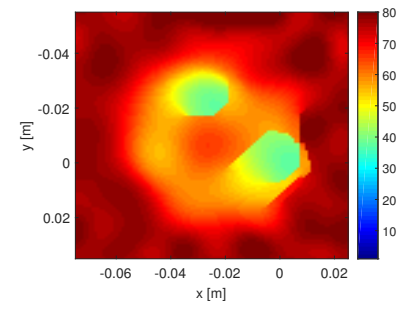

(c) $\operatorname{Re}\left(\epsilon_{r}\right)$, MR-CSI

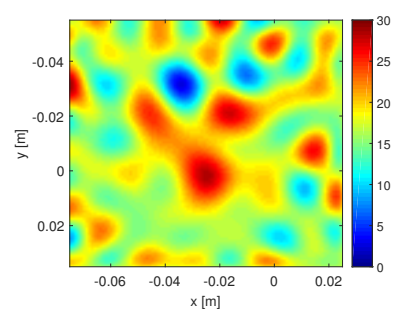

(b) $-\operatorname{Im}\left(\epsilon_{r}\right)$, MR-GNI

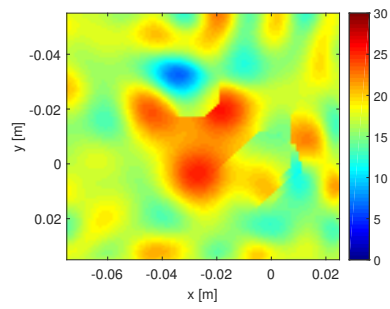

(d) $-\operatorname{Im}\left(\epsilon_{r}\right)$, MR-CSI
Fig. 3. Blind inversion of the human forearm data at $0.8 \mathrm{GHz}$ using the (a)-(b) MR-GNI and (c)-(d) MR-CSI algorithms. (Left and right columns represent the real and imaginary parts of the relative complex permittivity.)

the smaller dielectric cylinder within the larger one. Therefore, for this example, the edge-based SP approach outperforms the similarity-based SP approach. Finally, as it was the case with the previous example, the AMR-CSI still suffers from having some spurious edges. To alleviate this, we have also tried AMR-CSI (Type II) whose reconstruction is shown in Figure 2ff). As can be seen, we are able to fix the issue of spurious edges; however, due to the absence of $\left|\nabla \chi_{n}\right|^{2}$ in the denominator of (8), we do not get reconstruction with sharp edges within the larger cylinder and the reconstructed innercylinder permittivity undershoots its expected permittivity.

\section{B. Forearm Data Set}

This data set [16], [24] was collected from a human forearm at $0.8 \mathrm{GHz}$ using 24 co-resident dipole antennas immersed in a matching fluid (salt water) with the relative complex permittivity of about $77-j 17$. The blind inversion of this data set, which was originally reported in [16], [24], are presented in Figure 3 for the sake of comparison with inversion using $\mathrm{SP}{ }^{6}$ (The imaging domain has the size of $10 \times 9 \mathrm{~cm}^{2}$ discretized to $100 \times 100$ cells.) One item to note in the blind inversions is the fact that although the presence of the two bones are clear, their reconstructed relative permittivities are over-estimated. (According to [16], the relative complex permittivities of the bone and muscle are expected to be about $13-j 3$ and $56-j 20$ at the frequency of operation.)

In the next step, we utilize the image from the magnetic resonance imaging (MRI) of the forearm shown in Figure 4 (a) to create the SP shown in Figure $4(\mathrm{~b})$ as described in the

${ }^{6}$ Note that these blind inversions can still be improved by prescaling the real and imaginary parts of the unknown contrast in the inversion process [16], [25]. However, in that case, the utilized prescaling factor should be considered as a different type of prior information. This is not considered herein. 


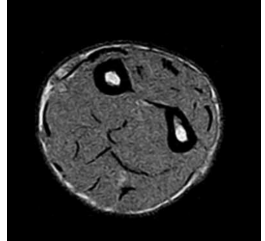

(a) MRI image

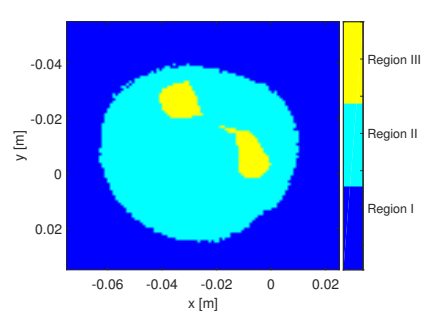

(b) $\mathrm{SP}$

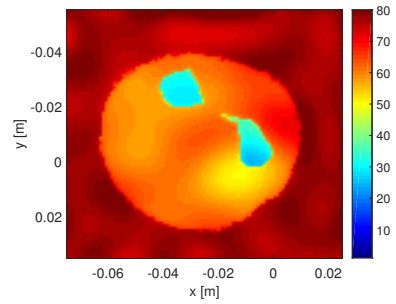

(d) $\operatorname{Re}\left(\epsilon_{r}\right)$, MRSP-GNI

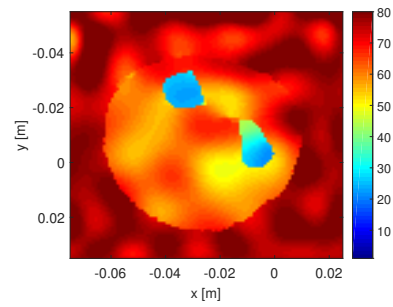

(f) $\operatorname{Re}\left(\epsilon_{r}\right)$, AMR-GNI

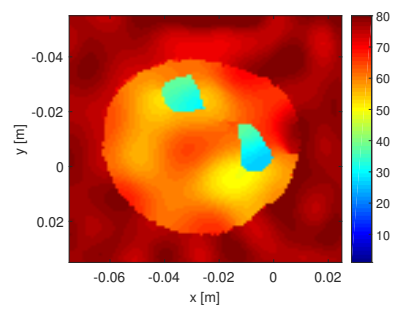

(h) $\operatorname{Re}\left(\epsilon_{r}\right)$, AMR-CSI

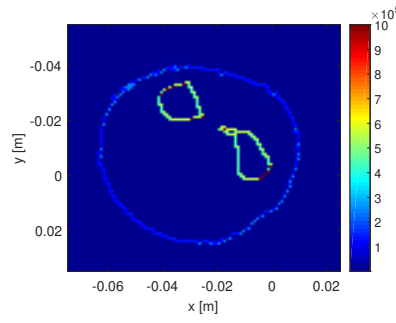

(c) $|\nabla P|^{2}$

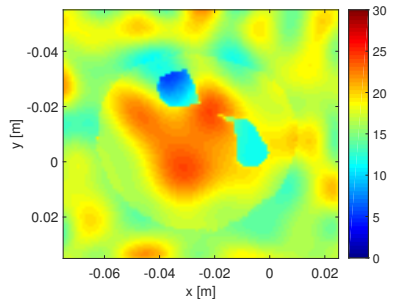

(e) $-\operatorname{Im}\left(\epsilon_{r}\right)$, MRSP-GNI

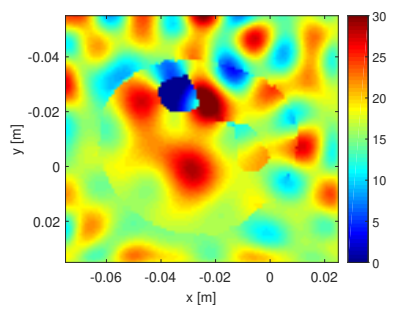

(g) $-\operatorname{Im}\left(\epsilon_{r}\right)$, AMR-GNI

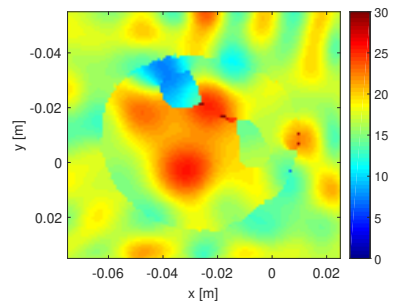

(i) $-\operatorname{Im}\left(\epsilon_{r}\right)$, AMR-CSI
Fig. 4. (a) The MRI image of the forearm (This image was taken from [16], C [2013] IEEE, and was also presented at [24|). (b) The SP derived from the MRI image. (c) The SP in the form of preferred edges. Real [left] and imaginary [right] parts of the reconstructed relative complex permittivity using the (d)-(e) MRSP-GNI, (f)-(g) AMR-GNI, (h)-(i) AMR-CSI algorithms.

Appendix. The SP in the form of preferred edges is shown in Figure $4(\mathrm{c})$. The reconstruction of the real and imaginary parts of the relative complex permittivity using the SP by the MRSP-GNI, AMR-GNI, and AMR-CSI algorithms are shown in Figures 4(d)-(i). (Since the reconstruction using AMR-CSI (Type II) was similar to AMR-CSI reconstruction,

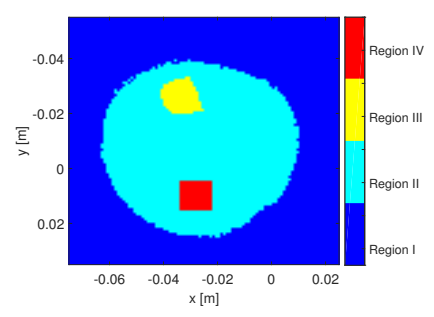

(a) Partial and wrong SP

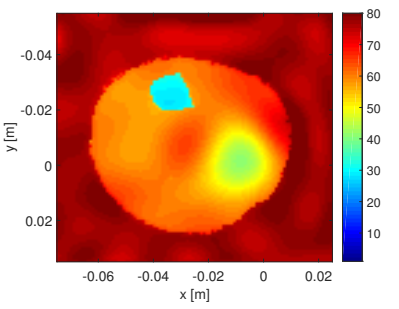

(c) $\operatorname{Re}\left(\epsilon_{r}\right)$, MRSP-GNI

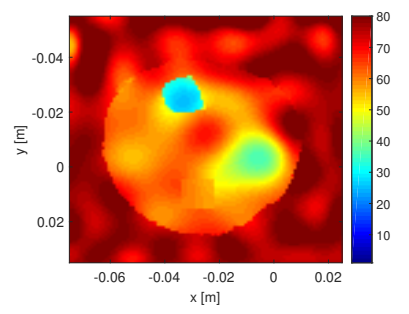

(e) $\operatorname{Re}\left(\epsilon_{r}\right)$, AMR-GNI

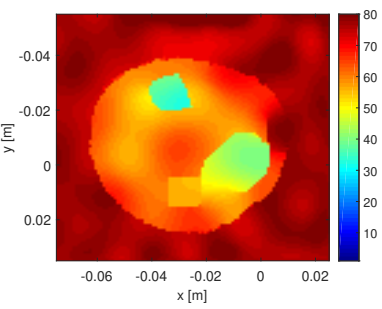

(g) $\operatorname{Re}\left(\epsilon_{r}\right)$, AMR-CSI

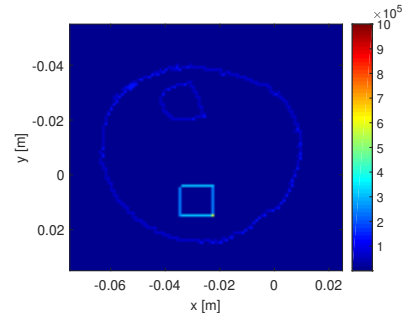

(b) $|\nabla P|^{2}$

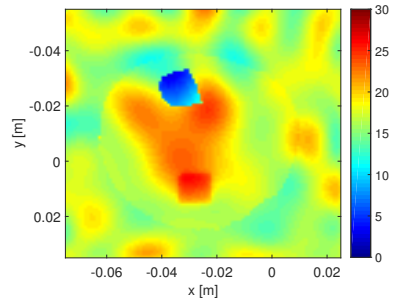

(d) $-\operatorname{Im}\left(\epsilon_{r}\right)$, MRSP-GNI

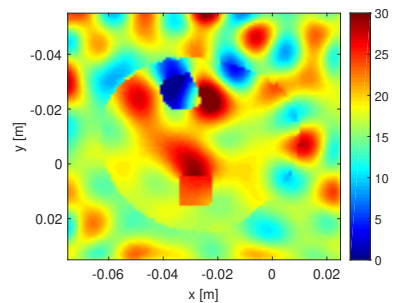

(f) $-\operatorname{Im}\left(\epsilon_{r}\right)$, AMR-GNI

(h) $-\operatorname{Im}\left(\epsilon_{r}\right)$, AMR-CSI

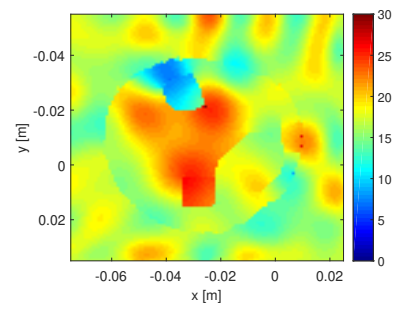

Fig. 5. (a) The incomplete (partial) and wrong SP which misses a bone region and mistakenly assume a square-shaped region. (b) SP in the form of edges $\left(|\nabla P|^{2}\right)$ to be given to the AMR-based approaches. Real [left] and imaginary [right] parts of the reconstructed relative complex permittivity using the (c)-(d) MRSP-GNI, (e)-(f) AMR-GNI, and (g)-(h) AMR-CSI algorithms.

it was not shown here for brevity.) As can be seen, the main improvement over the blind inversion is the enhanced quantitative retrieval of the bone's real-part permittivity. Other than this, the rest of the reconstruction does not show any significant improvements over blind inversion. Also, note that the MRSP-GNI reconstruction outperforms the AMR-GNI and AMR-CSI reconstrctions. This can be attributed to the fact that MRSP-GNI, due its similarity-based approach, has smoothed out the permittivity values within each region of the imaging domain. Finally, we consider to use the partial and wrong SP shown in Figure 5(a) and (b). The SP is partial as it misses one bone region; and, it is wrong as it mistakenly assumes a square-shaped region (Region IV in Figure 5 a)). 
Also, note the missing edges for one bone region as well as the wrong square edges in Figure 5(b). The MRSP-GNI, AMRGNI and AMR-CSI reconstructions using these SP are shown in Figures 5(c)-(h). As can be seen, all of these algorithms are able to detect the presence of the second bone which was missing in the partial SP. Also, they have all alleviated the presence of the wrong spatial prior regarding the squareshaped region. Note that although the square-shaped region is still visible in the reconstructions, its quantitative value is similar to the muscle region as it should.

\section{CONCLUSiON}

A simple and easy-to-implement approach to modify the existing microwave imaging algorithms employing MR was proposed to incorporate complete or partial available spatial priors into the inversion process. This method adds one extra term to the integrand of the standard multiplicative regularizer. Based on the results presented, the incorporation of spatial priors seems to enhance the quantitative accuracy achievable from microwave imaging.

\section{ACKNOWLEDGMENT}

We'd like to thank the Electromagnetic Imaging Laboratory at the University of Manitoba (Prof. Joe LoVetri) for letting us use the forearm data set, and also the Institut Fresnel for providing the freely-available experimental data. We'd also like to thank Prof. Jean-Charles Bolomey for his suggestion regarding the use of the MRI image and the forearm data set.

\section{APPENDIX}

Image registration needs to be performed so that we can use the MRI image for inversion. Herein, we have used a preliminary and ad hoc method to extract the SP from the MRI image. First, after applying some thresholding on the MRI image, its resolution was decreased using the MATLAB command interpn to make its discretization the same as that of the microwave inversion. Then, we have compared this postprocessed MRI image with the blind reconstruction shown in Figure 3. Based on the visual inspection, we have performed some translational movements (i.e., movements along the $\hat{x}$ and $\hat{y}$ directions) on the post-processed MRI image to merely adjust its overall external contour with respect to that of the blind reconstructions. We have then used this translated postprocessed MRI image as the SP.

\section{REFERENCES}

[1] P. M. Meaney, A. H. Golnabi, N. R. Epstein, S. D. Geimer, M. W. Fanning, J. B. Weaver, and K. D. Paulsen, "Integration of microwave tomography with magnetic resonance for improved breast imaging," Medical Physics, vol. 40, no. 10, p. 103101, 2013.

[2] H. Jiang, C. Li, D. Pearlstone, and L. Fajardo, "Ultrasound-guided microwave imaging of breast cancer: tissue phantom and pilot clinical experiments," Medical Physics, vol. 32, no. 8, pp. 2528-2535, 2005.

[3] N. Bayat, P. Mojabi, P. Mojabi, and J. LoVetri, "On Microwave Breast Imaging with Ultrasound Spatial Priors," 22020. [Online]. Available: https://www.techrxiv.org/articles/On_Microwave_ Breast_Imaging_with_Ultrasound_Spatial_Priors/11833095

[4] G. Boverman, C. Davis, S. Geimer, and P. M. Meaney, "Image registration for microwave tomography of the breast using priors from nonsimultaneous previous magnetic resonance images," IEEE J. Electromagn., RF, Microw. Med. Biol., vol. 2, no. 1, pp. 2-9, 2018.
[5] X. Song, M. Li, F. Yang, S. Xu, and A. Abubakar, "Three-dimensional joint inversion of EM and acoustic data based on contrast source inversion," IEEE J. Multiscale Multiphys. Comput. Tech., pp. 1-1, 2020.

[6] W. Hu, A. Abubakar, and T. M. Habashy, "Joint electromagnetic and seismic inversion using structural constraints," Geophysics, vol. 74, no. 6, pp. R99-R109, 112009.

[7] A. H. Golnabi, P. M. Meaney, and K. D. Paulsen, "Tomographic microwave imaging with incorporated prior spatial information," IEEE Transactions on Microwave Theory and Techniques, vol. 61, no. 5, pp. 2129-2136, May 2013.

[8] _ - "3D microwave tomography of the breast using prior anatomical information," Medical Physics, vol. 43, no. 4, pp. 1933-1944, 2016.

[9] A. H. Golnabi, P. M. Meaney, S. D. Geimer, and K. D. Paulsen, "3D microwave tomography using the soft prior regularization technique: Evaluation in anatomically realistic MRI-derived numerical breast phantoms," IEEE Trans. Biomed. Eng., vol. 66, no. 9, pp. 2566-2575, Sep. 2019.

[10] L. M. Neira, B. D. V. Veen, and S. C. Hagness, "High-resolution microwave breast imaging using a 3-D inverse scattering algorithm with a variable-strength spatial prior constraint," IEEE Transactions on Antennas and Propagation, vol. 65, no. 11, pp. 6002-6014, Nov 2017.

[11] M. Omer, P. Mojabi, D. Kurrant, J. LoVetri, and E. Fear, "Proof-ofconcept of the incorporation of ultrasound-derived structural information into microwave radar imaging," IEEE Journal on Multiscale and Multiphysics Computational Techniques, vol. 3, pp. 129-139, 2018.

[12] M. T. Bevacqua, R. Scapaticci, G. G. Bellizzi, T. Isernia, and L. Crocco, "Permittivity and conductivity estimation of biological scenarios via 3D microwave tomography," in 13th European Conference on Antennas and Propagation (EuCAP), March 2019, pp. 1-3.

[13] N. Abdollahi, D. Kurrant, P. Mojabi, M. Omer, E. Fear, and J. LoVetri, "Incorporation of ultrasonic prior information for improving quantitative microwave imaging of breast," IEEE Journal on Multiscale and Multiphysics Computational Techniques, vol. 4, pp. 98-110, 2019.

[14] N. Bayat and P. Mojabi, "Incorporating spatial priors in microwave imaging via multiplicative regularization," IEEE Transactions on Antennas and Propagation, vol. 68, no. 2, pp. 1107-1118, 2020.

[15] M. Bevacqua, G. Bellizzi, T. Isernia, and L. Crocco, "A method for effective permittivity and conductivity mapping of biological scenarios via segmented contrast source inversion," Progress in Electromagnetic Research, vol. 164, 2019.

[16] M. Ostadrahimi, P. Mojabi, A. Zakaria, J. LoVetri, and L. Shafai, "Enhancement of Gauss-Newton inversion method for biological tissue imaging," IEEE Transactions on Microwave Theory and Techniques, vol. 61, no. 9, pp. 3424-3434, Sept 2013.

[17] A. Abubakar, P. M. van den Berg, and J. J. Mallorqui, "Imaging of biomedical data using a multiplicative regularized contrast source inversion method," IEEE Trans. Microwave Theory Tech., vol. 50, no. 7, pp. 1761-1777, July 2002.

[18] A. Abubakar, P. M. van den Berg, T. M. Habashy, and H. Braunisch, "A multiplicative regularization approach for deblurring problems," IEEE Trans. Image Processing, vol. 13, no. 11, pp. 1524-1532, Nov 2004.

[19] P. Mojabi and J. LoVetri, "Overview and classification of some regularization techniques for the Gauss-Newton inversion method applied to inverse scattering problems," IEEE Transactions on Antennas and Propagation, vol. 57, no. 9, pp. 2658-2665, Sept 2009.

[20] P. Mojabi, "Investigation and development of algorithms and techniques for microwave tomography," Ph.D. dissertation, University of Manitoba, Winnipeg, Manitoba, Canada, 2010.

[21] X. Song, M. Li, F. Yang, S. Xu, and A. Abubakar, "Study on joint inversion algorithm of acoustic and electromagnetic data in biomedical imaging," IEEE J. Multiscale Multiphys. Comput. Tech., vol. 4, pp. 2-11, 2019.

[22] J.-M. Geffrin, P. Sabouroux, and C. Eyraud, "Free space experimental scattering database continuation: experimental set-up and measurement precision," Inverse Probl., vol. 21, pp. S117-S130, 2005.

[23] P. Mojabi and N. Bayat, "A multiplicative regularizer to incorporate prior spatial data in microwave imaging reconstruction," in European Conf. on Antennas and Propagation (EuCAP), March 2019, pp. 1-5.

[24] C. Gilmore, A. Zakaria, S. Pistorius, and J. LoVetri, "Microwave imaging of human forearms: Pilot study and image enhancement," International Journal of Biomedical Imaging, 2013.

[25] P. Mojabi and J. LoVetri, "A prescaled multiplicative regularized GaussNewton inversion," IEEE Trans. Antennas Propag., vol. 59, no. 8, pp. 2954-2963, 2011. 